\title{
Effects of Yerba Maté ingestion on fat utilisation during prolonged moderate intensity exercise
}

\author{
A. Alkhatib ${ }^{1,2}$ and R. Atcheson ${ }^{2}$ \\ ${ }^{1}$ Sport Science Program Department, College of Arts and Sciences, Qatar University, Doha, Qatar, 2713, Qatar and \\ ${ }^{2}$ Academy of Sport and Physical Activity, Sheffield Hallam University, Sheffield, Sheffield, S10 2BP, UK
}

Yerba Maté (YM), the plant of (Illex Paraguariensis) is widely ingested for its potential health benefits including anti-atherogenic and weight loss properties ${ }^{(1)}$. We have previously shown an exercise intensity-dependent effect of acute YM ingestion on fatty acid oxidation (FAO), particularly at low and moderate exercise intensity domains, determined using an incremental exercise protocol ${ }^{(2)}$. While, the augmented fat metabolism during those intensities implies improved exercise-metabolic weight-loss outcomes, the effects during prolonged exercise at this intensity range are unknown. This study aims to investigate the YM ingestion affects fat oxidation at prolonged moderate aerobic exercise intensities. We hypothesize that YM increase FAO during aerobic moderate intensity steady-state exercise.

Following the institutional approval, eleven healthy females (Mean \pm SD, age $=30 \cdot 8 \pm 7 \cdot 3$ years, stature: $167 \cdot 2 \pm 3 \cdot 9$, body mass: $61.5 \pm 2.8$ ) followed an initial incremental cardiopulmonary assessment ( $25 \mathrm{~W}$ increased every 3 min until exhaustion) to determine their peak oxygen uptake $\left(\mathrm{V}^{\circ} \mathrm{O}_{2}\right.$ peak $)$ and the range of exercise intensities corresponding to the cross-over point (COP), defined as the intensity or power output at which energy expenditure from carbohydrate predominates over that from fat sources ${ }^{(3)}$. This intensity range is known to be effective for exercise-dependent weight-loss ${ }^{(3,4)}$. Participants were randomised to ingest either $2 \mathrm{~g}$ of YM or placebo capsules (PLC), in a double-blinded, placebo controlled, repeated measures design. Following the ingestion participants rested for $120 \mathrm{~min}$ rest before ergometry cycling constantly for $30 \mathrm{~min}$ at an intensity corresponding to their COP. FAO and energy

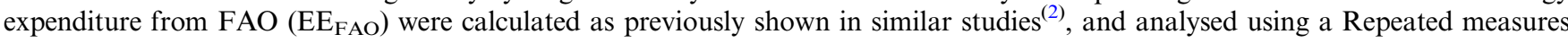
ANOVA design.

The average COP intensity corresponded to (Mean $\pm \mathrm{SD}, 37 \cdot 3 \pm 8 \cdot 3 \%$, relative to $V \cdot O_{2}$ peak at a power output of (50.6 $\pm 23 \cdot 4 \mathrm{~W}$ ). At this power or intensity, it was found that YM significantly increased FAO across all time points compared with PLC (Table 1).

Table 1. Fatty Acid Oxidation rate (FAO) during $30 \mathrm{~min}$ of constant load exercise in the YM vs. PLC (p $<0 \cdot 05$, main ANOVA effect), all data presented as (Mean \pm SD).

\begin{tabular}{|c|c|c|c|c|c|c|}
\hline Exercise Time (min) & 5 & 10 & 15 & 20 & 25 & 30 \\
\hline FAO-YM (g.min $\left.{ }^{-1}\right)$ & $0 \cdot 23 \pm 0 \cdot 08$ & $0 \cdot 19 \pm 0 \cdot 06$ & $0.19 \pm 0.06$ & $0 \cdot 21 \pm 0.07$ & $0.22 \pm 0.07$ & $0.23 \pm 0.08$ \\
\hline FAO-PLC $\left(\mathrm{g} \cdot \mathrm{min}^{-1}\right)$ & $0.21 \pm 0.07$ & $0 \cdot 15 \pm 0 \cdot 06$ & $0 \cdot 15 \pm 0 \cdot 05$ & $0 \cdot 17 \pm 0 \cdot 05$ & $0.17 \pm 0.06$ & $0.19 \pm 0.06$ \\
\hline
\end{tabular}

Acute ingestion of yerba mate can increase fat oxidation during moderate steady state intensity exercise, especially when the exercise is prescribed according to the individual's "fat-burning" intensities.

Heck CI, de Mejia EG (2007) J Food Sci 72(9): R138-51.

Alkhatib A (2014) Nut Metab 11(1): 42.

Brooks GA, Mercier J (1994) J Appl Physiol 76(6): 2253-61.

Venables MC, Jeukendrup AE (2008) Med Sci Sports Exerc 40(3):495-502. 